\title{
Raman Spectrum-Based Diagnosis Strategy for Bladder Tumor
}

\author{
Zhenghong Liu ${ }^{a, b}$ Pu Zhang ${ }^{b}$ Heng Wang ${ }^{b}$ Bin Zheng $^{a, b}$ Li Sun $^{c}$ \\ Dahong Zhang ${ }^{b}$ Jinhai Fand
}

aThe Second Clinical Medical College, Zhejiang Chinese Medical University, Hangzhou, China; bepartment of Urology, Zhejiang Provincial People's Hospital, People's Hospital of Hangzhou Medical College, Hangzhou, China; 'Hangzhou Medical College, Hangzhou, China; d Department of Urology, First Affiliated Hospital of Medical School, Xi'an Jiaotong University, Xi'an, China

\section{Keywords}

Raman spectrum · Bladder tumor $\cdot$ Diagnosis

\section{Abstract}

Raman spectroscopy is an optical technique that can potentially serve as a molecular diagnosis method. This approach is excellent in many aspects for diagnosing bladder tumors, and over the last 20 years, there has been a rapid increase in the number of related studies. However, no review article has covered the wide use of Raman spectroscopy in bladder tumors. A total of 26 original studies have suggested that Raman spectroscopy shows good performance in diagnosing bladder tumors from 4 aspects, including tissue sections, endoscopic methods, cell screening, and biomarkers. However, Raman spectroscopy needs to be modified by combining it with other techniques, and studies based on a large population are still urgently needed to expand its clinical value.

(c) 2021 S. Karger AG, Basel

\section{Introduction}

Raman scattering is an inelastic scattering laser light event, and the vibrational modes of molecules can be reflected explicitly by the shifts in energy of the scattered light, which allows for "fingerprint" recognitions of molecules [1]. The application of Raman spectroscopy (RS) holds great promise for the molecular analysis of diseases. Several advantages have become evident as RS has been applied in clinical practice. Unlike fluorescence, the instantaneous nature of Raman scattering makes it highly stable against photobleaching [1]. Its narrow peak width (approximately 10-100 times narrower than fluorescence peaks) produces an independent signal output of a single type of molecule without interference from other components, thus exhibiting excellent potential for multiplexing analysis. Moreover, in contrast to infrared spectroscopy, the interference from water is negligible in RS [2].

However, the inherently weak Raman scattering signal (one in one hundred million incident photons) prevents 
it from being used in clinical practice [1]. To further exploit the potential value of RS, new derivative techniques have been developed, including coherent anti-Stokes RS, stimulated RS, and surface-enhanced RS. These techniques have been explained in detail elsewhere [3]. Herein, the application of RS on bladder tumors is comprehensively summarized, and different situations where RS is fitted into various clinical uses are discussed.

\section{Tissue Section}

RS is mostly studied in tissue sections to reveal the differences in the sample components. Jong et al. [4] reported the first use of RS for observing bladders. Different layers of the bladder walls were distinguishable in vitro. The urothelium, muscle layer, and lamina propria featured a specific spectrum of fatty acids, actin, and collagen, respectively. In vivo, RS was obtained to evaluate the total tissue composition. This work highlighted the practical value of RS for visualizing the bladder. However, signal variation could result from variation in the bladder thickness when it was filled to different volumes. Furthermore, diversity in the urine spectrum was inevitable, which will be discussed later. All these uncertainties hampered the further development of standard spectra for normal bladders.

The application of RS was then extended to sections of bladder tissue for pathological diagnosis [5]. Microscopybased RS was used in combination with principal component fed and linear-discriminant analysis (PCA/LDA) to differentiate between normal, inflammatory, and malignant bladder pathologies in vitro. Initial outcomes from the 75 bladder samples indicated its efficiency in differentiating malignant bladder tumors from normal bladder and cystitis. In addition, RS also performed well in categorizing transitional cell carcinoma as high or low grade and noninvasive or invasive. However, the value of PCA/LDA was limited when small sample data sets were involved, and on this occasion, principal component analysis and support vector machine analysis (PCA/SVM) were more adaptable than PCA/LDA under the same conditions. Our group verified that RS could function in the diagnosis of bladder tumors when combined with PCA/SVM [6]. The RS of 8 normal bladder tissues and 15 bladder tumor tissues was studied, from which a sensitivity of $86.7 \%$ and a specificity of $87.5 \%$ were obtained. Another superior statistical method is partial least squares discriminant analysis (PLS/DA), which can provide additional information on group affinity and maximize the variations between samples [7]. A hierarchical classification based on PLS/
DA was initially used to differentiate tumors from nontumors, and it demonstrated a sensitivity of $92 \%$ and a specificity of $93 \%$, and afterward, it was used to predict the main differences between low-grade tumors and highgrade tumors and had a sensitivity of $85 \%$ [8]. The RS revealed the overall signal, which was collected from a relatively large tissue area. To gain precise information that was comparable to that of pathology diagnosis, detailed Raman spectroscopic maps were introduced [9]. The heterogeneous nature of the tumor lesion and normal tissue could be clearly monitored through scanning. Moreover, a spectrum cluster was defined as a category that covered spectral information for similar biochemical components, and this was normalized to the cluster-averaged spectrum for the tumor-related or nontumor-related components and essential elements in the pseudocolor Raman map. On the one hand, a fiber optic Raman spectroscope is more flexible than microscope-based RS for clinical practice, especially for in vivo use [10]. On the other hand, RS maintains the ability to identify benign and malignant pathological findings in the bladder. While RS has intriguing capabilities, individually it can cover only a specific aspect of diagnostics. Therefore, only a combination of multiple modalities can have the full diagnostic potential. Placzek et al. [11] reported the use of fiber optic-based optical coherence tomography for taking cross-sectional images of the bladder wall to diagnose for nonmuscle-invasive bladder cancer with a sensitivity of $78 \%$ and a specificity of $69 \%$. Meanwhile, RS was combined with it to discriminate the pathological grade by analyzing the featured lipid and collagen bands, and it provided a sensitivity of $81 \%$ and a specificity of $61 \%$. Enrico Baria reported the application of a compact system involving multimodal fiber optic-based spectroscopy, where RS, fluorescence, and reflectance spectra were collected together. A considerable increase in the diagnostic accuracy $(\geq 95 \%)$ of tumor grading and staging was achieved with the multimodal approach. Considering the complex clinical situation in which the multimodality imaging technique was applied, the contrast agent could compromise the specificity of RS. The use of 5-aminolevulinic acid increased the sensitivity of detecting bladder tumors due to the preferred accumulation of protoporphyrin IX in tumor cells $[12,13]$. Grimbergen et al. [14] reported that the presence of 5-aminolevulinic acid decreased the intensities of the Raman spectrum. However, the function of discriminating between malignant and benign tissues was preserved, indicating the possibility of combining RS with fluorescenceguided cystoscopy to improve the specificity of diagnosing bladder tumors. 


\section{Endoscope}

Endoscopic techniques to diagnose bladder tumors have been developed in recent years. However, RS has the shortest history in terms of clinical use among all types of endoscopic techniques. Draga et al. [15] attempted to achieve an in vivo diagnosis by high-volume RS, and they obtained a sensitivity of $85 \%$ and a specificity of $79 \%$. However, the relatively low specificity was due to out-offocus signal interference. Thus, RS might not function well in situations where specificity is required to be high, especially for the diagnosis of CIS. CIS, which is composed of only one cell layer, is a special type of bladder tumor. It demands a more precise sampling depth that is limited to approximately $15 \mu \mathrm{m}$ for RS. Currently, photodynamic diagnosis is the most sensitive endoscopic method for the diagnosis of CIS; however, its specificity ranges from 41.4 to $98.5 \%$, which is questionable [16]. The role of RS and its comparison to photodynamic diagnosis in the diagnosis of CIS have not been well documented, with only 2 cases that were mentioned in 1 study [17]. Theoretically, reducing the sampling depth could promise less confounding signals that are caused by more in-depth, noncancerous tissue layers. Confocal RS has a sampling depth of $<300 \mu \mathrm{m}$, which illuminates a thinner layer and generates less error. It was shown to gain a high specificity (100\%) without impeding its sensitivity (86\%) [18]. Confocal RS has great potential for improving specificity compared with other endoscopic methods, although CIS samples were not included in this study. However, all these RS techniques have the defect of an inherently weak signal. To compensate, high-power irradiation with long exposure times was needed; however, these modifications were clinically unacceptable. The amplification of RS intensity could be addressed through surface-enhanced Raman scattering (SERS). Functional SERS contrast agentassisted endoscopy showed superiority over these nonSERS endoscopes, with the aid of a maximum laser power of $42 \mathrm{MW}$ with 300-ms integration times [19]. More importantly, multiplexed molecular imaging gave it great potential for analyzing molecules of interest in the tumor region, thus improving specificity and sensitivity. Considering the complex pharmacokinetics and toxicity issues that occur as SERS contrast agents are intravenously administered, intravesical instillation is more acceptable when the SERS contrast agents are combined with a cystoscope [20]. Furthermore, the size, morphology, and surface chemistry of the agents should be delicately designed for effectively penetrating the bladder barrier and tissue [21]. However, a recent study indicated that nanoparticles without targeting ability could passively bind to bladder tumors when intravesical administration was conducted [22]. This could be caused by the disruption of the protective urothelial layer in the tumor region [23]. Another issue is that the spectra obtained from the SERS contrast agents should have little overlap with the spectra obtained from the dominant endogenous biomolecules in order to produce a background-free signal [24].

The use of RS-based ureteroscopy is also encouraged in the diagnosis of upper tract urothelial tumors. However, compared with cystoscopy, the limited operating space of ureteroscopy can affect the field of vision, and the type of TCC may be more biased toward CIS or smaller tumors. Hence, CIS could be diagnosed in confocal form by RS-based ureteroscopy, which overcomes the small space and low-resolution diagnosis that occurs with labeled probes. Regrettably, complete research on RS-based ureteroscopy is still lacking, which is also a direction we will need to study in the future.

\section{Cell Screening}

Urinary cytology is the only recommended noninvasive test for bladder tumors, and it is characterized by high specificity and low sensitivity [25]. Another concern is that the cytologic interpretation is user dependent. RS has entered the field of cell screening to establish a standard diagnosis model. Usually, the different spectral patterns of normal bladder cells and tumor cells can be visualized because they have distinct molecular profiles. High DNA and lipid concentrations and low protein concentrations are common features of tumor cells [26]. Meanwhile, the spatial changes of the phosphate group in the DNA structure serve as another recognition target, evidenced by the increased peak intensity at 1,095 $\mathrm{cm}^{-1}$ that corresponds to the contribution of the $\mathrm{PO}_{2}$ backbone stretching of DNA [27]. SVHUC-1 and MGH$\mathrm{U} 1$ are currently the most frequently studied cell models for comparison $[26,28]$. As SVHUC-1 and MGH-U1 cells were exposed to urine, a notable change in the RS spectrum could be observed over time, indicating that the conditions of sample collection were important for the application of RS in clinical urine samples [29]. Since the prostate and the bladder share the same access to excreted exfoliative cells, cross-contamination may occur if bladder tumors and prostate tumors coexist. Harvey et al. [30] applied Raman tweezers, referred to as Raman spectra plus optical tweezers, to trap, analyze, and differentiate PC-3 cells (prostate tumor cells) from MGH-U1 
cells. In addition, the Raman tweezer could also discriminate MGH-U1 from PC-3. The original RS generated from the samples containing a few bladder tumor cells was unobservable due to the autofluorescence background, but the SERS substrate with Ag nanocap arrays developed by our group could eliminate fluorescent interference and improve the SERS-relevant signal-tonoise ratio [31]. Jin et al. [32] adopted a strategy to incubate gold nanoparticles with bladder tumor cells. The gold nanoparticles could be either internalized into cells or attached to cells, and they served as SERS substrates to amplify the Raman signal. However, different types of cells exhibit different nanoparticle uptake efficiencies [33]. Therefore, it is difficult to quantitatively analyze the variance in SERS intensities among different cell lines. Shapiro et al. [34] reported that the Raman band at 1,584 $\mathrm{cm}^{-1}$ was a characteristic peak for bladder tumors, and the height of this peak correlated well with the tumor grade. It is encouraging to see a giant leap in the sensitivity $(92 \%)$ with a high specificity (91\%). A subsequent study indicated that the Raman band near $1,584 \mathrm{~cm}^{-1}$ originated from erythrocytes, and that the decrease in glycogen level and increase in fatty acid levels could work as efficient spectral markers for high-grade cancerous cells [35]. Another reliable spectral marker was the large nucleus to cytoplasm ratio, which could be monitored for the preselection of urothelial cells through coherent anti-Stokes RS.

\section{Biomarkers}

Recent interest in the noninvasive screening test has promoted a trend in specific bladder tumor markers [36]. However, the principal components in urine are varied and blended which leads to an inability to make an accurate diagnosis for bladder tumors. To detect different molecular signatures in urine that are related to bladder tumor and other genitourinary tract pathologies, Huttanus et al. [37] developed RS-based chemometric urinalysis (Rametrix) as a direct method for screening liquid urine. Using a model built with 22 principal components, BCA was detected with $80.4 \%$ accuracy, $82.4 \%$ sensitivity, and $79.5 \%$ specificity. Although label-free RS behaved well when distinguishing normal subjects and bladder patients, it was eclipsed by the labeled RS when it was combined with specific bladder tumor markers [38]. Bladder tumor markers have clinical significance, and they are responsible for the screening processes, early diagnosis, surveillance, staging, and prognosis predic- tion [36]. Multimarker analysis could further increase the sensitivity and specificity, which could fit into the multiplexed RS function [36]. Another reason to be concerned was that the variation of the label-free RS spectrum of urine samples resulted from the diversity of urine composition, concentration, and $\mathrm{pH}$ among males [39]. In contrast, labeled RS could achieve quality control and relatively good reproducibility. Kong et al. [40] introduced a metal carbonyl-functionalized BMFON substrate into a rapid immunoassay for the urine test, and alpha-1 antitrypsin was chosen as the biomarker. Although it was claimed to be a label-free SERS detection, metal carbonyl, which was the Raman reporter, determined the nature of the so-called "labeled RS." This SERS-based bioassay exhibited advantages, including the avoidance of the prior urine sample purification step and the low sampling collection volume $(10 \mu \mathrm{L})$. Nevertheless, the sensitivity was relatively limited compared to ELISA. Feng et al. [41] developed a dual-mode nanoprobe for detecting telomerase activity based on SERS and colorimetry. Two Au nanoparticles with a diameter of $13 \mathrm{~nm}$ were gathered as dimers in the absence of telomerase, and they presented a purple color and an enhanced SERS signal due to the hot spots formed at the narrow gaps of the dimers. When telomerase was present, it triggered DNA primer extension with the repeat units to form the extended sequence, which replaced the single DNA strand anchored to the $\mathrm{Au}$ nanoparticle, which then led to the disaggregation of dimers. Consequently, the color changed from purple to red, and the SERS signal was reduced. The initial outcome in 12 patients proved its clinical value. Nevertheless, only the SERS spectrum could produce an effective response when a low cell ratio was present in urine samples. Our group proposed a molecular beacon that was based on an SERS nanoprobe, which could specifically respond to the presence of a bladder tumor biomarker (survivin mRNA) [42]. The signal switch could be triggered both in fluorescence and SERS modalities. Binding features in the SERS modality showed better sensitivity (limit of detection: $0.194 \mathrm{pmol}$ ) and specificity. Thus, in 13 patients, the SERS modality was more acceptable than the fluorescence modality for initial clinical use. The sensitivity for bladder tumors was $84.6 \%$, and it dropped to $80 \%$ only if early-stage bladder tumors ( $\leq \mathrm{T} 1)$ were considered. There was some uncertainty about the stability of the SERS signal even though it showed excellent sensitivity. For example, the SERS signal changed with different nanoscale dimensions under different temperatures. Temperature could affect the nanoscale dimension, leading to a change 
in the SERS signal [43]. The method proposed by Si et al. [44] standardized the unstable signal produced by reactive nanoprobes by introducing another nonreactive nanoprobe. Hyaluronic acid (HA), with unique physical properties, is a newly discovered biomarker for bladder cancer. Gold nanorods (AuNRs) were first functionalized with 4-thiobenzonitrile (TBN) as the Raman reporter (TBN-AuNRs), and the 4-thiophenylacetylene-functionalized gold-silver alloy nanoparticles (TPA-AuAgNPs) acted as the reference. HA is a hyaluronidase (HAase)-recognizing component. In the presence of HA, the TBN-AuNRs aggregated due to the strong electrostatic interaction between the positively charged TBNAuNRs and negatively charged HA, which produced hot spots and greatly enhanced the Raman signal of TBN. Once HAase was present, HA was digested into small fragments with weaker negative charges, and thus the TBN-AuNRs regained good dispersion with faded SERS signals. During the whole process, the TPA-AuAgNPs maintained their monomers due to the inertia of HAase, and thus the process was suitable for standardization. The normalized signal intensity of $I_{1,974} / I_{2,220}$ showed a linear correlation with the concentration of HAase in the range of $5.00 \pm 0.21-70.00 \pm 0.39 \mathrm{U} \mathrm{mL}^{-1}$. However, there is an intrinsic difference between the physiochemical properties of TBN-AuNRs and TPA-AuAgNPs, which determines the different responses to uncontrollable variates. Hence, the standardization reference should be equipped with the same reactive nanoprobe form. Our group developed dual-molecular-beacon-labeled SERS nanoprobes of which one molecular beacon recognized GAPDH mRNA and acted as the standardization reference, which coexisted on the surface of Au nanourchins with another molecular beacon [42]. Extracellular vesicles have been adopted as urogenital tumor biomarkers in recent studies, and they can also be used to establish new diagnostic approaches for bladder tumors [45]. With biomolecular cargo that is reflective of the cell of origin, extracellular vesicles provide abundant bioinformation for multiomics diagnosis [45]. Zhang et al. [46] applied an SERS nanoprobe to evaluate the phenotype of bladder cancer cell line (C3) EVs by identifying 3 surface protein biomarkers, which were glypican-1, epithelial cell adhesion molecules, and the CD44 variant isoform 6 . Herein, 3 SERS nanotags were prepared by functionalizing AuNPs with different Raman reporter molecules (DTNB, MBA, or TFMBA) and different antibodies (MIL38, epithelial cell adhesion molecule, or CD44 variant isoform 6) and incubating them in a filtered, conditioned EV-suspended medium. By incubating with mag-

Raman Spectrum-Based Diagnosis

Strategy for Bladder Tumor netic bead-CD63 antibodies and inducing magnetic separation, the relative abundance of surface protein biomarkers could be quantified by monitoring the SERS signal of their corresponding Raman reporters. This method was sensitive and effective, and the nanotags were prepared in a single test without complicated isolation steps. It was also measured to a concentration of 2.3 $\times 10^{6}$ particles $/ \mathrm{mL}$, which shows a higher multiplexing capability than most other reported EV profiling techniques. Nonetheless, having a mass of magnetic beads theoretically leads to uncontrollable gaps, and ultimately some biomarkers cannot be counted. When nanoprobes are assembled on the surface of EVs, the true expression level of the surface proteins might also be impaired by steric effects.

\section{Conclusion}

The wide application of RS in the bladder was presented in this article, and it demonstrates that RS has excellent potential for in vitro and in vivo diagnosis in clinical practice. Labeled and label-free RS techniques offer flexibility in different clinical situations. A prominent feature of RS is that it provides abundant information on the molecular components. However, applying RS to clinical reports is still challenging due to the time-consuming scanning procedure. Therefore, RS has been modified to be used in more advanced modalities, including coherent anti-Stokes RS, stimulated RS, and surface-enhanced RS. RS has also been developed for endoscopic use, such as fiber optic RS. An unexpected obstacle has been the shortage of research on urine markers for bladder tumors and RS. The high sensitivity and specificity of RS characterize it as a method for analyzing the molecule of interest, and because of the abilities it exhibits, the use of RS should be encouraged for urine marker screening. Another critical issue is that RS should be combined with other spectroscopic techniques to form a multimodality platform. To yield better diagnostic outcomes, more modalities should be used in the clinic. RS combined with fluorescence cystoscopy has been investigated but not fully exploited. An RS-based multimodality platform shows a promising future for clinical applications.

\section{Conflict of Interest Statement}

The authors have no conflicts of interest to declare. 


\section{Funding Sources}

This study was funded by the Medical Technology Plan of Zhejiang Province (2021KY042), China, and the Natural Science Foundation of Zhejiang Province (LQ21H160041), China.

\section{Author Contributions}

Z.L. and P.Z. contributed to writing/editing and data collection or management; H.W., B.Z., and L.S. contributed to suggestions and data proofreading; D.Z. and J.F. gave approval of the final version of this work; Z.L. and P.Z. contributed to the work equally and should be regarded as joint first authors; D.Z. and J.F. should be regarded as joint corresponding authors. All authors read and approved the final manuscript.

\section{References}

1 Raman CV, Krishnan KS. A new type of secondary radiation. Nature. 1928;121(3048): $501-2$.

2 Lane LA, Qian X, Nie S. SERS nanoparticles in medicine: from label-free detection to spectroscopic tagging. Chem Rev. 2015;115: 10489-529.

3 Kong K, Kendall C, Stone N, Notingher I. Raman spectroscopy for medical diagnostics: from in vitro biofluid assays to in vivo cancer detection. Adv Drug Deliv Rev. 2015;89:12134.

4 De Jong BW, Bakker Schut TC, Wolffenbuttel KP, Nijman JM, Kok DJ, Puppels GJ. Identification of bladder wall layers by Raman spectroscopy. J Urol. 2002;168:1771-8.

5 Crow P, Uff JS, Farmer JA, Wright MP, Stone $\mathrm{N}$. The use of Raman spectroscopy to identify and characterize transitional cell carcinoma in vitro. BJU Int. 2004;93(9):1232-6.

6 Wang L, Fan JH, Guan ZF, Liu Y, Zeng J, He DL, et al. [Study on bladder cancer tissues with Raman spectroscopy]. Guang Pu Xue Yu Guang Pu Fen Xi. 2012;32:123-6.

7 Liu W, Sun Z, Chen J, Jing C. Raman spectroscopy in colorectal cancer diagnostics: comparison of PCA-LDA and PLS-DA models. J Spectrosc. 2016;2016(2):1.

8 Cordero E, Rüger J, Marti D, Mondol AS, Hasselager T, Mogensen K, et al. Bladder tissue characterization using probe-based Raman spectroscopy: evaluation of tissue heterogeneity and influence on the model prediction. J Biophotonics. 2020;13(2): e201960025.

9 De Jong BW, Schut TC, Maquelin K, Van Der Kwast T, Bangma CH, Kok DJ, et al. Discrimination between nontumor bladder tissue and tumor by Raman spectroscopy. Anal Chem. 2006;78(22):7761-9.

10 Crow P, Molckovsky A, Stone N, Uff J, Wilson $\mathrm{B}$, Wongkeesong LM. Assessment of fiberoptic near-infrared raman spectroscopy for diagnosis of bladder and prostate cancer. Urology. 2005;65(6):1126-30.

11 Placzek F, Cordero Bautista E, Kretschmer S, Wurster LM, Knorr F, González-Cerdas G, et al. Morpho-molecular ex vivo detection and grading of non-muscle-invasive bladder cancer using forward imaging probe based multimodal optical coherence tomography and Raman spectroscopy. Analyst. 2020;145(4): 1445-56.
12 Riedl CR, Daniltchenko D, Koenig F, Simak $\mathrm{R}$, Loening SA, Pflueger H. Fluorescence endoscopy with 5-aminolevulinic acid reduces early recurrence rate in superficial bladder cancer. J Urol. 2001;165:1121-3.

13 Krieg RC, Fickweiler S, Wolfbeis OS, Knuechel R. Cell-type Specific protoporphyrin IX metabolism in human bladder cancer in vitro. Photochem Photobiol. 2000;72(2): 226.

14 Grimbergen MC, van Swol CF, van Moorselaar RJ, Uff J, Mahadevan-Jansen A, Stone N. Raman spectroscopy of bladder tissue in the presence of 5-aminolevulinic acid. J Photochem Photobiol B. 2009;95(3):170-6.

15 Draga RO, Grimbergen MC, Vijverberg PL, van Swol CF, Jonges TG, Kummer JA, et al. In vivo bladder cancer diagnosis by high-volume Raman spectroscopy. Anal Chem. 2010; 82(14):5993-9.

16 Jocham D, Stepp H, Waidelich R. Photo dynamic diagnosis in urology: state-of-the-art. Eur Urol. 2008;53:1138-50.

17 Crow P, Uff JS, Farmer JA, Wright MP, Stone $\mathrm{N}$. The use of Raman spectroscopy to identify and characterize transitional cell carcinoma in vitro. BJU Int. 2004 Jun;93(9):1232-6.

18 Barman I, Dingari NC, Singh GP, Kumar R, Lang S, Nabi G. Selective sampling using confocal Raman spectroscopy provides enhanced specificity for urinary bladder cancer diagnosis. Anal Bioanal Chem. 2012;404(10):30919.

19 Zavaleta CL, Garai E, Liu JT, Sensarn S, Mandella MJ, Van De Sompel D, et al. A Ramanbased endoscopic strategy for multiplexed molecular imaging. Proc Natl Acad Sci U S A. 2013;110:E2288-97.

20 Hoshyar N, Gray S, Han H, Bao G. The effect of nanoparticle size on in vivo pharmacokinetics and cellular interaction. Nanomedicine. 2016;11(6):673-92.

21 Ramesh N, Memarzadeh B, Ge Y, Frey D, VanRoey M, Rojas V, et al. Identification of pretreatment agents to enhance adenovirus infection of bladder epithelium. Mol Ther. 2004;10(4):697-705

22 Davis RM, Kiss B, Trivedi DR, Metzner TJ, Liao JC, Gambhir SS. Surface-enhanced Raman scattering nanoparticles for multiplexed imaging of bladder cancer tissue permeability and molecular phenotype. ACS Nano. 2018; 12:9669-79.
23 Pan Y, Volkmer JP, Mach KE, Rouse RV, Liu JJ, Sahoo D, et al. Endoscopic molecular imaging of human bladder cancer using a CD47 antibody. Sci Transl Med. 2014;6:260ra148260.

24 Yin Y, Li Q, Ma S, Liu H, Dong B, Yang J, et al. Prussian blue as a highly sensitive and background-free resonant Raman reporter. Anal Chem. 2017;89(3):1551-7.

25 Babjuk M, Böhle A, Burger M, Capoun O, Cohen $\mathrm{D}$, Compérat EM, et al. EAU guidelines on non-muscle-invasive urothelial carcinoma of the bladder: update 2016. Eur Urol. 2017;71(3):447-61.

26 Canetta E, Riches A, Borger E, Herrington S, Dholakia K, Adya AK. Discrimination of bladder cancer cells from normal urothelial cells with high specificity and sensitivity: combined application of atomic force microscopy and modulated Raman spectroscopy. Acta Biomater. 2014;10(5):2043-55.

27 Dovbeshko GI, Gridina NY, Kruglova EB, Pashchuk OP. FTIR spectroscopy studies of nucleic acid damage. Talanta. 2000;53(1): 233-46.

28 Praveen BB, Mazilu M, Marchington RF, Herrington CS, Riches A, Dholakia K. Optimisation of wavelength modulated Raman spectroscopy: towards high throughput cell screening. PLoS One. 2013;8(6):e67211-8.

29 Canetta E, Mazilu M, De Luca AC, Carruthers AE, Dholakia K, Neilson S, et al. Modulated Raman spectroscopy for enhanced identification of bladder tumor cells in urine samples. J Biomed Opt. 2011;16(3):037002.

30 Harvey TJ, Faria EC, Henderson A, Gazi E, Ward AD, Clarke NW, et al. Spectral discrimination of live prostate and bladder cancer cell lines using Raman optical tweezers. J Biomed Opt. 2008;13(6):064004.

31 Liu Y, Huang LQ, Wang J, Tong HM, Yuan L, Zhao LH, et al. [Fabrication of silver ordered nanoarrays SERS-active substrates and their applications in bladder cancer cells detection]. Guang Pu Xue Yu Guang Pu Fen Xi. 2012;32:386-90.

32 Jin D, Chen H, Cao M, Yang G, Xue W, Huang Y. SERS measurement of the bladder cancer cells with the nanoparticles. Pak J Pharm Sci. 2015;28:1853-6.

33 Dykman LA, Khlebtsov NG. Uptake of engineered gold nanoparticles into mammalian cells. Chem Rev. 2014;114(2):1258-88. 
34 Shapiro A, Gofrit ON, Pizov G, Cohen JK, Maier J. Raman molecular imaging: a novel spectroscopic technique for diagnosis of bladder cancer in urine specimens. Eur Urol. 2011;59(1):106-12.

35 Yosef HK, Krauss SD, Lechtonen T, Jütte H, Tannapfel A, Käfferlein HU, et al. Noninvasive diagnosis of high-grade urothelial carcinoma in urine by Raman spectral imaging. Anal Chem. 2017;89(12):6893-9.

36 Nezos A, Pissimisis N, Lembessis P, Sourla A, Dimopoulos P, Dimopoulos T, et al. Detection of circulating tumor cells in bladder cancer patients. Cancer Treat Rev. 2009;35(3): 272-9.

37 Huttanus HM, Vu T, Guruli G, Tracey A, Carswell W, Said N, et al. Raman chemometric urinalysis (Rametrix) as a screen for bladder cancer. PLoS One. 2020;15(8):e0237070.
38 Li S, Li L, Zeng Q, Zhang Y, Guo Z, Liu Z, et al. Characterization and noninvasive diagnosis of bladder cancer with serum surface enhanced Raman spectroscopy and genetic algorithms. Sci Rep. 2015;5:9582.

39 Bonifacio A, Cervo S, Sergo V. Label-free surface-enhanced Raman spectroscopy of biofluids: fundamental aspects and diagnostic applications. Anal Bioanal Chem. 2015;407(27): 8265-77.

40 Kong KV, Leong WK, Lam Z, Gong T, Goh D, Lau WK, et al. A rapid and label-free SERS detection method for biomarkers in clinical biofluids. Small. 2014;10:5030-4.

41 Feng E, Zheng T, Tian Y. Dual-mode Au nanoprobe based on surface enhancement Raman scattering and colorimetry for sensitive determination of telomerase activity both in cell extracts and in the urine of patients. ACS Sens. 2019;4(1):211-7.

42 Zhang P, Zhang Y, Liu W, Cui D, Zhao X, Song J, et al. A Molecular beacon based surface-enhanced Raman scattering nanotag for noninvasive diagnosis of bladder cancer. J Biomed Nanotechnol. 2019;15(7):1589-97.
43 Ma L, Wang J, Huang H, Zhang Z, Li X, Fan Y. Simultaneous thermal stability and ultrahigh sensitivity of heterojunction SERS substrates. Nanomaterials. 2019;9(6):1-10.

44 Si Y, Li L, He B, Li J. A novel surface-enhanced Raman scattering-based ratiometric approach for detection of hyaluronidase in urine. Talanta. 2020;215:120915.

45 Nawaz M, Camussi G, Valadi H, Nazarenko I, Ekström K, Wang X, et al. The emerging role of extracellular vesicles as biomarkers for urogenital cancers. Nat Rev Urol. 2014;11(12): 688-701.

46 Zhang W, Jiang L, Diefenbach RJ, Campbell $\mathrm{DH}$, Walsh BJ, Packer NH, et al. Enabling sensitive phenotypic profiling of cancer-derived small extracellular vesicles using surface-enhanced Raman spectroscopy nanotags. ACS Sens. 2020;5(3):764-71. 